\title{
DAKAR, UN PUERTO AL SERVICIO DEL IMPERIO: INFRAESTRUCTURAS, INSTITUCIONES Y ADMINISTRACIÓN PORTUARIA EN ÁFRICA OCCIDENTAL
} (1857-1939)

\section{DAKAR, UM PORTO A SERVIÇO DO IMPÉRIO: INFRAESTRUTURAS, INSTITUIÇÕES E ADMINISTRAÇÃO PỎTUÁRIA NA ÁFRICA OCIDENTAL (1857-1939) \\ DAKAR, A PORT AT THE SERVICE OF AN EMPIRE: INFRASTRUCTURES, INSTITUTIONS AND PORT MANAGEMENT IN WEST AFRICA (1857-1939)}

\section{DAKAR, UN PORT AU SERVICE DE L'EMPIRE : INFRASTRUCTURES, INSTITUTIONS ET ADMINISTRATION PORTUAIRES EN AFRIQUE OCCIDENTALE (1857-1939)}

达卡尔，为帝国服务的港口：在西部非洲的基础建设，机构设置港务管理 (1857-1939)

DOI: 10.5533/1984-2503-20146104

\section{Daniel Castillo Hidalgo ${ }^{1}$}

\section{RESUMEN}

Los puertos coloniales en África Occidental jugaron un papel decisivo en la introducción de estos territorios en los circuitos internacionales de materias primas. El puerto de Dakar (Senegal) centralizó el comercio exterior regional, convirtiéndose en la principal infraestructura comercial del África Occidental Francés. Esta importancia se plasmó en la concentración de inversiones en la mejora de las infraestructuras portuarias, así como en el desarrollo de las conexiones intermodales hacia el hinterland, dónde se desarrollaban los cultivos de exportación. Este proceso de modernización continua de las infraestructuras portuarias tuvo un reflejo en el crecimiento de la actividad comercial en la región, así como la orientación de la economía productiva hacia el sector exportador de commodities coloniales. En esta comunicación se presentan las principales fases desarrolladas en la modernización de las infraestructuras portuarias en Dakar, observando además su relación constante con el crecimiento de la actividad comercial y la importancia regional creciente de la metrópoli oeste-africana. Además, se analizarán las principales instituciones y el modelo de administración portuaria, estrechamente ligados con los intereses metropolitanos y el estatus jurídico de Senegal como colonia.

\footnotetext{
${ }^{1}$ Investigador Post-doctoral. Departamento de Ciencias Históricas. Universidad de Las Palmas de Gran Canaria (España).

E-mail: dcastillohidalgo@gmail.com
} 
Palabras clave: Puertos, África Occidental, Instituciones, Administración portuaria, globalización.

\section{RESUMO}

Os portos coloniais na África Ocidental desempenharam um papel decisivo na introdução destes territórios nos circuitos internacionais de matérias-primas. O porto de Dakar (Senegal) centralizou o comércio exterior regional, se tornando a principal infraestrutura comercial da África Ocidental Francesa. Esta importância foi refletida na concentração de investimentos na melhoria da infraestrutura portuária, assim como no desenvolvimento de conexões intermodais para o interior, onde se desenvolveram as culturas de exportação. Este processo de modernização contínua da infraestrutura portuária refletia-se no crescimento da atividade comercial na região, bem como na orientação da economia produtiva para o setor de exportação de commodities coloniais. Este artigo apresenta as principais fases desenvolvidas na modernização das infraestruturas portuárias, em Dakar, observando também sua relação com o crescimento da atividade empresarial e a ascendente importância regional da metrópole do Oeste africano. Além disto, serão analisadas as principais instituições e o modelo de administração portuária relacionados aos interesses metropolitanos e ao estatuto jurídico do Senegal como colônia.

Palavras-chave: Portos, África Ocidental, instituições, administração portuária, globalização.

\section{ABSTRACT}

West Africa's colonial ports played a decisive role in introducing these territories to international raw-material trade routes. The port of Dakar in Senegal centralized regional foreign trade, going on to become the main trade hub of French West Africa. This importance was reflected in the investments poured into improving the port's infrastructure, as well as in the construction of transport links to the interior of the country, where the growing of crops for export was developed. This continual modernization of the port infrastructure was reflected in the growth of trade activities in the region, as well as in the orientation of the productive economy to the export sector of colonial commodities. This article outlines the main phases in the modernization of Dakar's port infrastructures, and also discusses its relationship with the growth of corporate activities and the increasing regional importance of the Western African metropolis. The article also analyses the main 
institutions and the port management model, closely related to metropolitan interests and the legal status of Senegal as a colony.

Key words: Ports, West Africa, institutions, port management, globalization.

\section{RÉSUMÉ}

Les ports coloniaux d'Afrique occidentale ont joué un rôle décisif dans l'inclusion de ces territoires dans les circuits internationaux de matières premières. Le port de Dakar (Sénégal) a longtemps centralisé le commerce extérieur régional pour devenir la principale infrastructure commerciale de l'Afrique occidentale française. Cette importance est corroborée par la concentration des investissements pour l'amélioration des infrastructures portuaires, ainsi que par le développement de connexions intermodales avec le reste du pays, où se développèrent les cultures d'exportation. Ce processus de modernisation continue des infrastructures portuaires a provoqué la croissance de l'activité commerciale dans la région, mais aussi l'orientation de l'économie vers le secteur des exportations de matières premières coloniales. Cette article présentera les principales phases de modernisation des infrastructures portuaires à Dakar et les mettra en relation avec la croissance des milieux d'affaires et de l'importance régionale de la métropole d'Afrique de l'Ouest. Seront également étudiées les principales institutions portuaires et leur administration, étroitement liées aux intérêts de la Métropole et au statut juridique du Sénégal en tant que colonie.

Mots-clés : Ports, Afrique occidentale, institutions, administration portuaire, mondialisation.

\section{摘要}

西部非洲的殖民地港口在推进该地区的国际原材料流通中起了决定性作用。塞内加尔的达卡 尔港集中了这个地区的对外贸易, 成为西非法属殖民地的商贸中心。其重要地位可以从对这 个港口的基建投资, 向内地扩展的路运, 水运的发展, 内地的主要功能就是生产出口农产品 。港口的基建连续不断的现代化伴随了当地的商业发展和当地出口大宗农产品。本论文分析 了达卡尔港的基建发展现代化的几个阶段, 基建与商贸企业活动的联系, 西非殖民地经济与 宗主国的矢系。另外还分析了主要机构的特征和港务管理模式, 这些都和宗主国的利益直接 关联，和塞内加尔的殖民地法律地位直接关联。

关键词：港 $\square$, 西非, 机构，港口管理，全球化 


\section{Introducción}

Este artículo analiza el modelo de administración portuaria y el proceso de modernización de las infraestructuras en el puerto colonial de Dakar (Senegal), un nodo de comunicaciones paradigmático en África Occidental ${ }^{2}$. Emplazado en la península de Cabo Verde, el puerto de Dakar fue la principal infraestructura de transportes para el Imperio francés en África Occidental, sirviendo como puerto de escala, pero sobre todo como base comercial (Gateway) para el África Occidental Francés (AOF). El puerto de Dakar se proyectó desde sus orígenes en 1857 como una base logística y militar para la expansión francesa hacia el interior del continente, pudiendo alcanzar el interior del Níger (actual Mali) y los territorios próximos a Sudán, para de este modo establecer el gran imperio territorial en África que se extendiera desde Argelia hasta el Golfo de Guinea (Mapa 1).

\section{Mapa 1. Localización geográfica del puerto de Dakar}

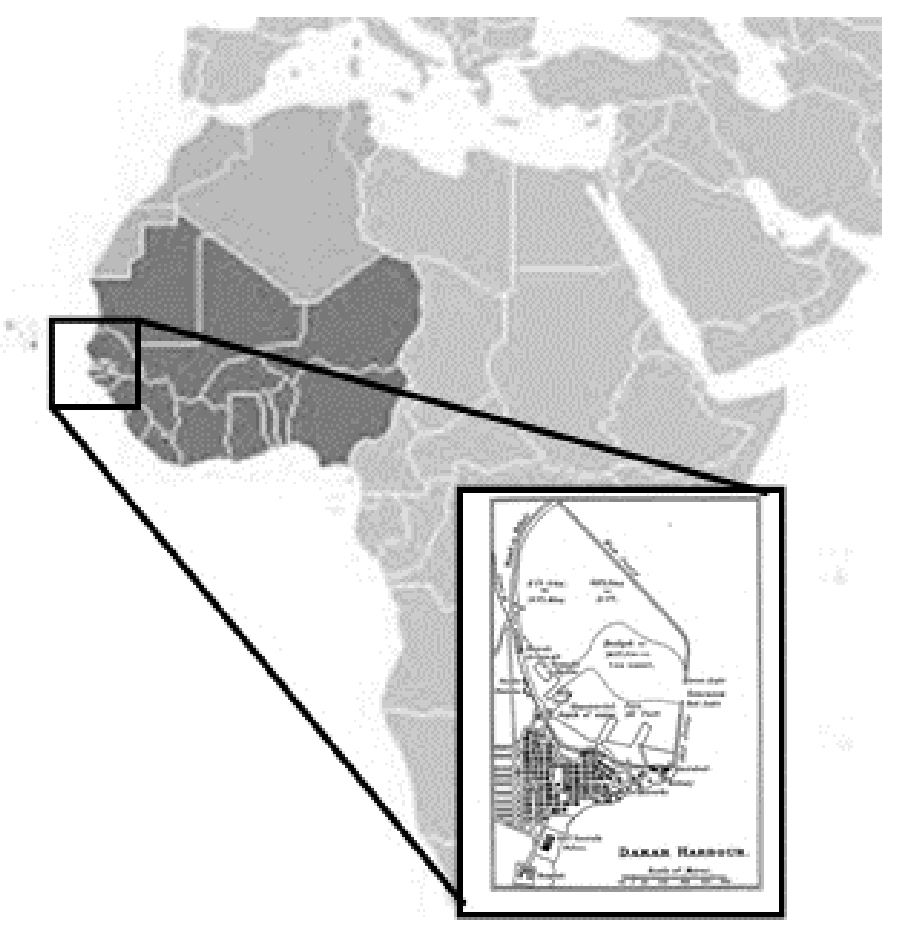

Fuente: Elaboración propia.

\footnotetext{
${ }^{2}$ Este artículo forma parte del Proyecto de I+D+l del Ministerio de Educación y Ciencia del Gobierno de España: Modelos de Gestión de Puertos y la Comunidad Portuaria en el Ámbito Atlántico (siglos XIX y XX) (HAR2010-17408). Una versión previa ha sido presentada en el V Congreso Internacional "La Gobernanza de los Puertos Atlánticos (Siglos XIV-XXI)" celebrado en Madrid, del 13 al 15 de junio de 2013.
} 
Para ello, la colaboración e interacción entre el puerto oceánico, el ferrocarril (en el último tercio del siglo XIX), las empresas imperiales y las fuerzas militares de ocupación y pacificación fue esencial. La combinación de estos agentes imperiales incentivó la extensión del capitalismo y sobre todo la reorganización del sistema productivo en los espacios coloniales, orientando su economía hacia una creciente mercantilización, al amparo de las doctrinas económicas liberales ${ }^{3}$. El primer reflejo en esta materia se plasmó en la expansión de los cultivos agrarios de exportación en Senegal como los cacahuetes, que dominaron y transformaron notablemente las estructuras socioeconómicas tras el establecimiento del sistema político colonial.

En este esquema de expansión imperial, el puerto de Dakar fue uno de sus elementos rectores, sirviendo primero como base logística para la importación de todo tipo de materiales de construcción, armamento y manufacturas metropolitanas, para posteriormente convertirse en el principal centro exportador para el AOF. Su importancia estratégica y su localización geográfica estructuraron las infraestructuras intermodales modernas (especialmente las conexiones de ferrocarril), capitalizando las conexiones interiores que permitían unas rápidas y eficaces comunicaciones entre la costa atlántica y el interior de Senegal y Níger, alcanzando las confluencias del río Níger y el Senegal hasta Tombouctou y Bamako en la primera década del siglo XX. Estas conexiones intermodales con inicio y final en el puerto de Dakar permitieron el crecimiento del comercio exterior, animando además a los productores africanos a incrementar sus esfuerzos en los cultivos de mercado que adquirían los intermediarios de las grandes compañías comerciales europeas para su distribución hacia Dakar, dónde eran exportadas hacia Europa y Estados Unidos. Por otra parte, desde 1910 el puerto de Dakar comenzó a configurarse como un puerto de escala al servicio de la navegación francesa, en concurrencia con los puertos canarios que dominaban esta función portuaria como consecuencia del paso preferente por las islas de la flota mercante británica que lideraba el comercio regional. No obstante, el puerto de Dakar también recibía las escalas de buques británicos, holandeses, belgas, nórdicos y en menor medida alemanes, hecho que quedó de relieve durante la Primera Guerra Mundial, cuando los buques británicos -tanto civiles como militares- reemplazaron las escalas canarias y de Cabo Verde por la mayor

\footnotetext{
${ }^{3}$ Debrie, Jean (2010). "From colonization to national territories in continental West Africa: the historical geography of a transport infrastructure network". In Journal of Transport Geography, 18, p. 292-300.
} 
seguridad frente a la guerra submarina alemana que proporcionaba la bahía de Dakar con respecto a los puertos insulares.

No obstante, el hecho distintivo de Dakar en la red de puertos de África Occidental es su carácter comercial, orientado especialmente al comercio exterior y la redistribución de las importaciones hacia el interior a través de las conexiones de ferrocarril ${ }^{4}$. Sintéticamente, el puerto de Dakar respondería a las características esenciales de un puerto Gateway (carrefour) debido a su estructura comercial, con unos indicadores portuarios entre los que destaca un notable comercio de importación y exportación ${ }^{5}$. Esta notable actividad económica, que comenzó a despuntar en las primeras décadas del siglo XX generó un proceso de concentración económica en la ciudad portuaria. Esto se reflejó en la creciente presencia de empresas comerciales, consignatarias, carboneras, aseguradoras, financieras, así como un incipiente sector hostelero y de servicios vinculado a los tráficos de escala. Por otra parte, el incremento de las funciones comerciales urbanas se sumó a una mayor presencia de instituciones políticas y reguladoras que incrementaron la importancia de Dakar como centro político, como por ejemplo la creación de la Cámara de Comercio (1888), la instalación de la Base naval y Arsenal en el puerto (1898), la apertura de la sede central del Banque d'Afrique Occidentale (1901), así como el traslado de la capital del AOF (1902) desde Saint-Louis, la apertura de oficinas consulares y embajadas o la dotación de tribunales y centros hospitalarios.

El incremento de la actividad económica asociada al formidable crecimiento del "comercio legítimo" entre 1880 y 1914 motivaron importantes reformas en las infraestructuras portuarias para hacerlas competitivas y eficientes, pero también impulsaron la reforma de la administración portuaria, en consonancia con los procesos de modernización portuaria que estaban desarrollándose en los principales nodos portuarios internacionales. Las necesidades de la navegación moderna requerían de profundas transformaciones en las instalaciones portuarias dónde se estaba desarrollando el comercio internacional y se estaba incrementando la competencia inter-portuaria. Esta competencia inter-portuaria adquiría una mayor relevancia en el caso de los puertos de escala cuya función dominante requería de unos servicios ágiles, eficaces y baratos en las tareas de carboneo, avituallamiento de alimentos y agua, así como en las

\footnotetext{
${ }^{4}$ Debrie, Jean (2012). "The West African port system : global insertion and regional particularities". In EchoGéo, 20 (avril/juin).

${ }^{5}$ Bird, James (1971). Seaports and seaport terminals, London: Hutchinson University Library.
} 
reparaciones navales y los servicios anexos a la actividad portuaria (seguros, banca, sector hostelero, etc.).

Sin embargo, la red portuaria de África Occidental comenzó a conformarse de tal modo que la competencia entre los diferentes puertos se centró especialmente en los servicios de escala, dadas las características de las estructuras comerciales en los puertos continentales, dominados por diferentes grados de proteccionismo colonial. En este aspecto, las diferentes conferencias y acuerdos internacionales como los de 1895 y 1898 trataron de regular y repartir el comercio en el Golfo de Guinea entre las diferentes potencias imperiales, afectando a los tráficos portuarios. En lo que se refiere a los servicios de escala, los puertos isleños de Canarias y Cabo Verde, fueron competidores entre ellos hasta 1910, cuando el puerto de Dakar (Senegal) comienza a desbancar la escala de San Vicente de Cabo Verde, agregando la oferta de servicios portuarios a sus funciones comerciales. El desarrollo de otros nodos portuarios entre 1910 y 1920 como Casablanca (Marruecos) o Freetown (Sierra Leona) que ofertaban también servicios portuarios de escala, incrementarían la complementariedad entre los diferentes puertos, comenzando a establecerse un modelo de jerarquía portuaria, fundamentada en las funciones desempeñadas y su creciente rol en la red portuaria que se estaba desarrollando ${ }^{6}$. Las funciones portuarias eran dominadas además por un conjunto de empresas interconectadas y que disponían de una red de agencias e intermediarios en los principales centros portuarios y comerciales de la costa occidental africana. A través de su capacidad financiera y su poder de mediación sobre las instituciones políticas, las grandes empresas europeas como Wilson and Sons, Elder Dempster o la Compagnie Française d'Afrique Occidentale (CFAO) desarrollaron una poderosa red comercial con presencia en los principales puertos oceánicos de África Occidental, sirviendo a la expansión imperialista europea ${ }^{7}$.

Este artículo ofrece un análisis en el largo plazo de la evolución de las infraestructuras y del modelo de administración portuaria en Dakar, observando los factores internos y externos que incidieron en la reforma portuaria entre 1857 y 1939 . En este periodo pueden designarse dos procesos integrales de reforma portuaria. El primero de ellos se produjo entre 1880 y 1910 , cuando comenzaron a implementarse elementos característicos de los puertos industriales modernos (implementación de la

\footnotetext{
${ }^{6}$ Polónia, Amelia (2010). "European seaports in the Early Modern Age: concepts, methodology and models of analysis". In Cahiers de la Méditerranée, 80, p. 17-39.

${ }^{7}$ Rodney, Walter (1971). How Europe Underdeveloped Africa, Dakar: Pambazuuka Press [2011].
} 
intermodalidad: ferrocarril-navegación; dragados y señalización de la zona portuaria, construcción de muelles, almacenes y astilleros). La segunda gran oleada de reformas puede trazarse entre 1920 y 1936, dónde se expande la actividad comercial, se crea el consejo de administración del puerto y se modifican las infraestructuras portuarias para adaptarlas a las necesidades de la navegación impulsada por fuel-oil. Por otra parte, este artículo analiza la contribución del puerto de Dakar a la expansión del capitalismo industrial en África Occidental debido a su carácter como eje vertebral del sistema comercial y de transportes en el AOF.

La primera parte de este artículo (1) analiza el proceso de modernización de las infraestructuras portuarias entre 1857 y 1939, los planes de reforma y cómo éstas modificaciones respondían a las necesidades de un comercio exterior en crecimiento. Del mismo modo, se observa la mayor interacción entre el transporte terrestre guiado en las infraestructuras portuarias desde la década de 1880. La segunda parte (2) se encarga del estudio de las instituciones portuarias y el modelo de administración en el puerto de Dakar, así como su evolución interna y su influencia sobre el desarrollo de la actividad comercial. Finalmente se ofrecen algunas conclusiones (3).

\section{El proceso de modernización de un puerto colonial africano (1857-1939)}

El puerto de Dakar es un claro ejemplo del funcionamiento de un puerto colonial oceánico en África Occidental. En líneas generales, estos puertos continentales eran la puerta de entrada y salida de mercancías para los territorios coloniales, capitalizando la mayor parte del comercio exterior. La estructura comercial en África durante el periodo colonial se fundamentó en las escasas relaciones interiores entre los diferentes territorios coloniales. El sistema productivo -extractivo- diseñado e impulsado por las instituciones imperiales se orientó hacia el exterior, con un diseño de las redes de transporte extrovertido, estructurado en torno a los grandes puertos oceánicos. Esta estructura se plasmó en unas redes de transporte terrestre lineales con una escasa influencia de las ramificaciones locales que permitieran una mayor integración territorial. No obstante, los puertos oceánicos fueron los elementos esenciales que permitieron la introducción de los territorios coloniales en la economía de mercado global que se desarrollaba de modo formidable en las últimas décadas del siglo XIX. Los puertos oceánicos oeste africanos como el de Dakar permitieron la evacuación de las producciones agrícolas y mineras así 
como el desarrollo de las economías de exportación y una mayor dependencia de estos territorios del comercio exterior y las fluctuaciones de los mercados internacionales.

La estructura económica diseñada por el Gobierno colonial en el AOF se estructuró en torno al papel capital y predominante de Dakar sobre el conjunto de la red portuaria senegalesa. Este proceso se intensificó en el primer tercio del siglo XX, cuando los viejos centros comerciales de Saint-Louis, Gorée y Rufisque, fueron sustituidos progresivamente por el incremento de la importancia de Dakar. La red de transportes de Senegal y el territorio del Níger y Sudán (actuales Burkina Faso y Mali) se diseñaron de tal manera que las conexiones ferroviarias tenían como objetivo alcanzar Dakar a través de las diferentes estaciones y escalas fluviales que habían dominado el comercio tradicional africano. Este protagonismo creciente se sustentó en la inversión en la mejora de las infraestructuras portuarias en Dakar, puesto que los puertos oceánicos de Rufisque, Gorée o Saint-Louis no ofrecían unas expectativas ni posibilidades de crecimiento debido esencialmente a diferentes condicionantes geográficos ${ }^{8}$. Los ingenieros como Émile Pinet Laprade y otros administradores coloniales planificaron el futuro de Senegal y el conjunto del AOF a través del desarrollo de las infraestructuras portuarias en la bahía de Dakar. Sin embargo, el puerto por sí mismo era insuficiente para un proyecto más amplio y complejo de explotación económica del Hinterland, para lo cual era imprescindible el establecimiento de un sistema de transportes moderno en cuanto las condiciones tecnológicas, económicas y militares lo permitieron, tal y como exponía Pinet-Laprade, capitán ingeniero destinado en Gorée en 1857:

Nous croyons donc pouvoir conclure d'une manière certaine que l'établissement d'un chemin de fer entre Sor et Dakar sera possible dans peu d'années et produira une révolution des plus heureuses dans la marche des opérations commerciales du Sénégal, tout en favorisant au plus haut degré le développement de nos établissements du Cap Vert (...) L'avenir de cette intéressante colonie dépendraitil davantage du petit mouvement que pourraient donner à son commerce de détail les bâtiments qui entrent dans le fleuve que de la grande facilité et de l'économie que l'on introduira dans ses rapports avec l'Europe?

El sistema tradicional de transportes en África Occidental aprovechaba los ríos como vías naturales de comercio, dónde se desarrollaba una fuerte actividad de intercambios de todo tipo. A ello se sumaba el tráfico terrestre de caravanas que tenían al

\footnotetext{
${ }^{8}$ Charpy, Jacques (2007). Dakar. Naissance d'une Métropole, Dakar : Editions Les Portes du Large. Véase también: Charpy, Jacques (2011). “Aux origines du port de Dakar”. In Outre-Mers, T.99-370/371, p. 301-317. ${ }^{9}$ Entrevista realizada a Pinet-Laprade, publicada en Les Colonies Françaises (1857), La Revue Coloniale, Imprimerie et Librairie Administrative de Paul Dupont, Paris, p. 10-11.
} 
camello y al burro como principal elemento de carga y movilidad. Sin embargo, la expansión del capitalismo industrial desplazó este comercio africano tradicional a un segundo plano, puesto que los avances e innovaciones propias de la revolución industrial, y especialmente el ferrocarril fueron los elementos capitales del proceso de expansión europea hacia el interior del continente, así como el desarrollo de los cultivos de exportación. Las conexiones de ferrocarril que se construyeron desde 1882 facilitó la introducción de estos territorios en los mercados internacionales de materias primas.

En cualquier caso, la inversión imperial en infraestructuras en el AOF se concentró en el puerto de Dakar, cuyas ramificaciones se extendieron hacia el Alto Senegal y Mali gracias a las conexiones ferroviarias que se iniciaron en 1882 con la primera sección de la línea Dakar-Saint Louis (DSL) que alcanzaba a Rufisque (1883). Esta conexión ferroviaria permitió que la producción del río Senegal pudiera ser fácilmente transportada desde la vieja capital de Senegal hacia el puerto moderno que estaba desarrollándose en Dakar. El transporte fluvial en Saint-Louis estaba protagonizado por transportistas africanos propietarios de canoas y otras embarcaciones pequeñas, así como por empresas europeas como Méssageries Africaines, utilizando vapores de escaso calado que permitían la navegación segura hasta el puerto fluvial de Kayes. Por otra parte, Rufisque fue el principal centro exportador de cacahuetes hacia Europa desde la década de 1880 hasta los años 1930, recibiendo los buques de cabotaje que transportaban la producción de cacahuetes, caucho, cera y otras commodities de los puertos fluviales como Kaolak en el Sine-Saloum, así como buena parte de la producción de Casamance. Rufisque sustituyó así al viejo entrepôt de Gorée, que no pudo adaptarse a las necesidades de la navegación y sucumbió a la pugna inter-portuaria frente a los puertos vecinos de Dakar y el citado Rufisque. Los buques de vela y pequeños vapores transportaban las mercancías desde Kaolack, Ziguinchor y la Casamance hacia Rufisque, dónde posteriormente eran re-embarcadas en Dakar y exportadas hacia Europa y otros puertos americanos. Una vez se completó la primera sección de la línea DSL en 1883 entre Dakar y Rufisque, el transporte de cacahuetes y otras mercancías comenzaron a realizarse a través de la línea de ferrocarril, disminuyendo el tiempo, las rupturas de carga los costes agregados. La otra gran conexión con influencia en Dakar se construyó en la primera década del siglo XX. La línea Kayes-Thiès (1903-09) permitió que Thiès (estación cercana a Dakar y con conexión directa en la línea DSL) alcanzara Kayes, que era el último puerto fluvial navegable en el río Senegal. A partir de ahí, la línea de Sudán (1881-1904) se extendía entre Koulikoro y 
Tombouctou en el interior de Mali. ${ }^{10} \mathrm{~A}$ lo largo de las diferentes líneas de ferrocarril se desarrollaron los principales cultivos de exportación y esencialmente el cultivo del cacahuete que se convirtió en la principal fuente de ingresos para los agricultores africanos del AOF.

Debe destacarse que toda esta red de comunicaciones terrestre tenía como eje el puerto de Dakar, que ha absorbido desde la década de 1930 no menos del ochenta por ciento del comercio exterior de Senegal. Este papel predominante sobre la actividad comercial regional requirió de un proceso de modernización de sus infraestructuras de manera continua y proporcional a las demandas tecnológicas. En lo que se refiere a la modernización de las infraestructuras y a la capacidad de proyección de las mismas, el único punto habilitado para la dotación de un puerto moderno en Senegal era la bahía de Dakar. Los antiguos puertos oceánicos de Gorée, Rufisque o Saint-Louis se vieron superados por las características geográficas de la bahía de Dakar protegida del oleaje y los vientos y cuyos fondos rocosos evitaban la formación de bancos de arena, que es una de las características de la costa occidental africana. La disposición de suelo para la construcción de almacenes y depósitos de carbón también fue otro de los elementos que potenciaron la capacidad de Dakar para albergar un puerto moderno al servicio de la navegación internacional (véase Gráfico 1).

\section{Gráfico 1. Factores de competitividad inter-portuaria}

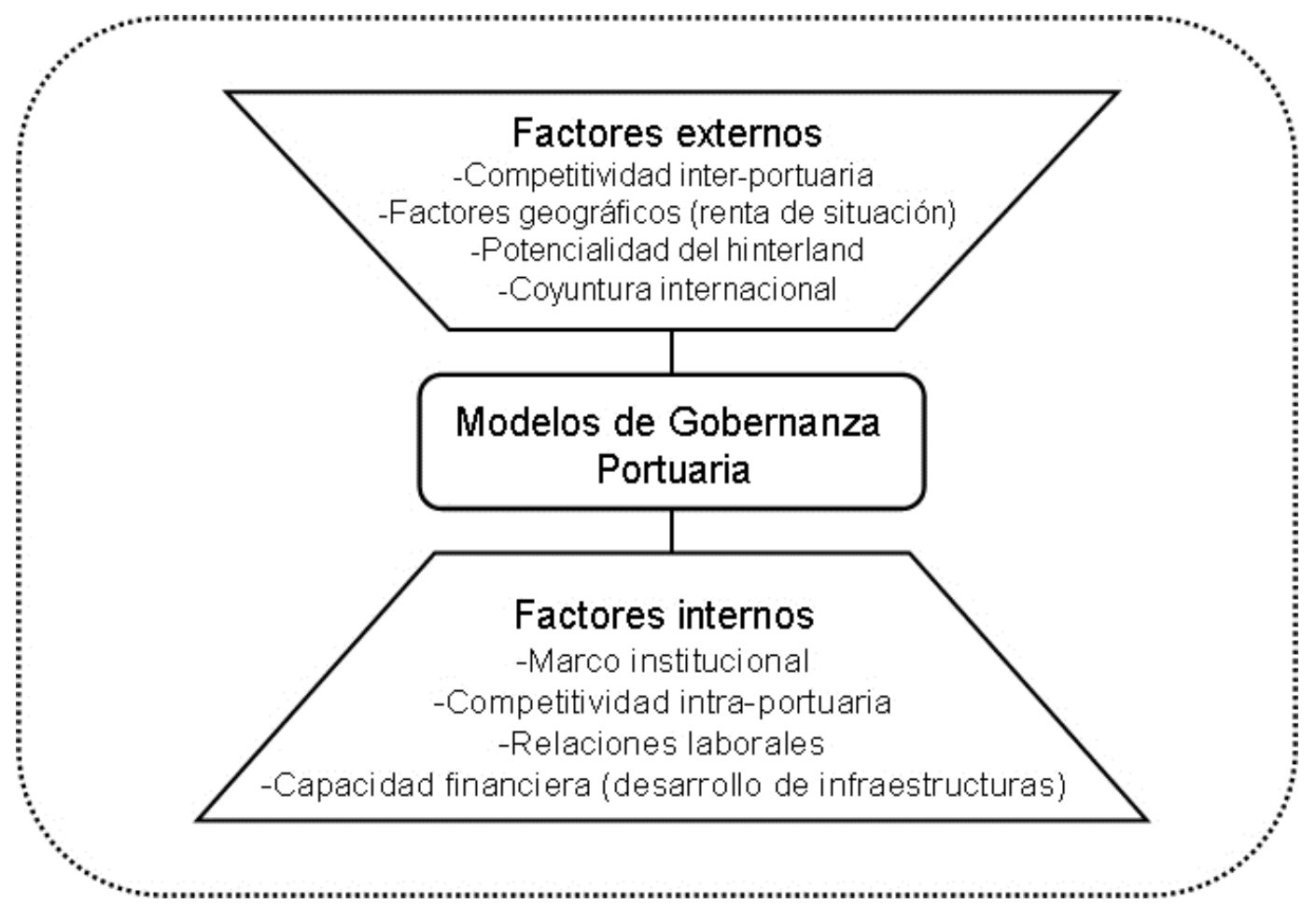

\footnotetext{
${ }^{10}$ Public Record Office (PRO) 879/86 (1914).
} 


\section{Fuente: Elaboración propia.}

En 1857, el Gobierno francés llegó a un acuerdo con la compañía de navegación girondina Méssageries Impériales para establecer una escala regular en la Bahía de Dakar en sus rutas entre Burdeos, Brasil y el río de La Plata. Este acuerdo a dos bandas junto con la toma en posesión de la península de Cabo Verde por parte francesa y el proyecto de expansión hacia el interior ejercieron como elementos dinamizadores de la creación del puerto. La negativa de la empresa a realizar la escala en Dakar por las malas condiciones del puerto de Gorée y las nulas expectativas comerciales provocaron que hasta 1866, los vapores de Méssageries hicieran sus escalas en el vecino puerto de Mindelo, en San Vicente (Cabo Verde), dónde existían empresas británicas de servicios portuarios británicos desde 1852. El crecimiento de la competencia comercial regional entre Gran Bretaña y Francia en estos años de expansión imperial por África Occidental motivó el interés por parte de París de establecer un programa de transportes en sus posesiones coloniales para mejorar las infraestructuras y adaptarlas a las crecientes demandas tecnológicas. Como ha sido señalado, Dakar se encontraba en el eje de esa política de modernización integral. ${ }^{11}$

Los primeros trabajos en el puerto de Dakar comenzaron con la construcción del dique sur (Petit Jetée) con el que se pretendían alcanzar los 200 metros y ampliar el área de aguas protegidas en la bahía. ${ }^{12}$ En Noviembre de 1866 el vapor -Le Guianne- hizo escala en Dakar, estableciendo el primer tráfico regular entre Burdeos, Dakar y Brasil. No sería hasta la década de 1880 cuando puede observarse un progreso más acentuado en la modernización de las infraestructuras portuarias. Entre 1880 y 1910 puede establecerse el primer periodo de reformas portuarias en Dakar, con la creación de muelles comerciales, la extensión de los diques, el dragado de los fondos y la introducción de la intermodalidad en las instalaciones portuarias a través de sistemas Decauville. La instalación de vías férreas en el puerto de Dakar mediante el sistema Decauville también se desarrolló en Rufisque desde la década de 1880, merced a los proyectos de modernización portuaria impulsados por el Ministro Fraycinet (1878) en el que se trató de mejorar la productividad de los puertos mediante un menor impacto en los procesos de fractura de carga (Marnot, 1999, 653). La extensión del sistema Decauville en Dakar se

\footnotetext{
${ }^{11}$ Consular and Diplomatic Reports (CDR). No.520. Report on French Colonies (1908).

${ }^{12}$ ANS. Puertos, Sección O. Carta de Chausselaup-Laubat, Ministro Francés de las Colonias al Gobernador de Senegal, 24 de Julio de 1863.
} 
desarrolló sobre todo en el periodo 1904-10 cuando se construyeron los muelles comerciales que facilitaban el embarque y desembarque de mercancías, reduciendo los tiempos de espera. Estas obras se complementaron con los trabajos realizados en la Base Naval (1896-98) dónde se construyó un dique seco, así como almacenes de carbón y edificios destinados a la custodia de material de guerra y los barracones para los soldados. En 1904, el gobierno Federal del AOF fue autorizado por parte del gobierno metropolitano a la contratación de un crédito de 65 millones de francos que debían ser invertidos en un proyecto integral de mejora de las infraestructuras. ${ }^{13} \mathrm{El}$ marco institucional de autonomía presupuestaria aprobada en 1900 para las colonias francesas requería de este tipo de negociaciones con París para las políticas que requerían un gran endeudamiento público. Sobre este préstamo de 65 millones, el montante destinado para las obras de remodelación del puerto de comercio de Dakar alcanzó los 40 millones (62 por ciento del total). La contratación de los trabajos en el puerto se sacó a concurso público, y los ganadores del mismo fueron las empresas francesas MM. Jammy et Galtier y $M M$. Hersent quiénes también habían participado en la construcción del Arsenal del puerto en 1898 y que luego intervendrían en las obras del puerto de Casablanca. ${ }^{14}$ Las obras de modernización integral del puerto de Dakar se desarrollaron en este proyecto entre 1904 y 1910 y se tradujeron en una reforma radical de la bahía con la extensión del dique sur (Grand Jetée) y la construcción de un dique en la zona norte que permitió el cierre y abrigo de la zona portuaria, mejorando la seguridad. Estos trabajos se complementaron con los dragados de los fondos rocosos de la bahía, alcanzando profundidades de hasta doce metros para permitir el desplazamiento seguro de los buques de mayor calado y tonelaje. Finalmente, se proyectaron y construyeron dos muelles comerciales en la zona sur del puerto que disponían de un sistema Decauville en los nuevos terraplenes anexos a la zona portuaria. En esos terraplenes debían construirse almacenes, oficinas, talleres y depósitos de carbón (Mapa 2). ${ }^{15}$

Con la inauguración del puerto de comercio en 1910, el tráfico portuario en Dakar se incrementó notablemente en la siguiente década hasta convertirse en la década de

\footnotetext{
${ }^{13}$ Marnot, Bruno (1999). "La politique des ports maritimes en France de 1860 à 1920". In Histoire, économie et société, 18:3, p. 653.

${ }_{14}$ Archives Nationales Section Outre-Mer (ANSOM). 14MIOM/1462, 24 de Diciembre de 1903. Véase tambien: Journal Officiel de la République Française, 20 de Enero de 1904. Los Archivos Británicos incluyen interesante información sobre estos empréstitos. Como muestra: PRO. Foreign Office 27/3682. Carta de HG.Mackie, cónsul británico en Dakar al Marques de Landowe, Ministro Británico del Foreign Office, 25 de Enero de 1904. Debe ser destacado que desde 1900 el AOF y el resto de territorios coloniales disponían de autonomía financiera para desarrollar sus propios presupuestos.

${ }^{15}$ ANSOM. Travaux Publics (TP). Dossier 21. Comité des Travaux Publics des Colonies (1898).
} 
1930 en el principal núcleo comercial de todo el AOF. Este incremento de su actividad en la década de 1930 se sustentó en las actuaciones desarrolladas en la década de 1920 como consecuencia del Plan Sarraut. No obstante, durante la Primera Guerra Mundial el puerto de Dakar había adquirido una importancia capital para los Aliados al reemplazar las escalas de los buques comerciales y militares de los puertos insulares de Cabo Verde y Canarias. Este incremento de su rol regional estratégico implicó una mejora en las instalaciones que se orientaron hacia los servicios de suministro de carbón. Para ellos se construyeron nuevos almacenes carboneros y se aumentó el stock de combustible disponible por parte de las empresas carboneras británicas que dominaban el mercado del carbón en África Occidental. ${ }^{16}$ Los stocks de carbón se aumentaron gracias al acuerdo adoptado en 1916 entre las empresas británicas Elder Dempster y Wilson and Sons, y las autoridades franco-británicas que deseaban poseer una base naval segura en la costa occidental africana ante la ofensiva submarina alemana. Estas dos grandes empresas que eran buques insignia de la expansión del capitalismo británico trasladaron sus stocks de carbón de otros puertos como La Plata (Argentina), Mindelo (Cabo Verde) y Las Palmas (Islas Canarias) para servir a la flota Aliada, transportando también parte de ese carbón hacia el puerto de Freetown (Sierra Leona), fortaleciendo la presencia de los Aliados en la región. ${ }^{17}$

\footnotetext{
${ }^{16}$ Esto incluía las oficinas de la Administración portuaria que se construyeron en esa década también. ANSOM. Travaux Publics. Carton 26. Dossier 2, Dossier 8 (1904).

17 Sobre este asunto, la documentación británica es remarcable: PRO, Ministry of Transports, MT23/564; MT10/1896 (1916). PRO. Admiralty ADM 1/9214 (1917).
} 
Mapa 2. El proyecto de modernización del puerto de comercio de Dakar (1904-1910)

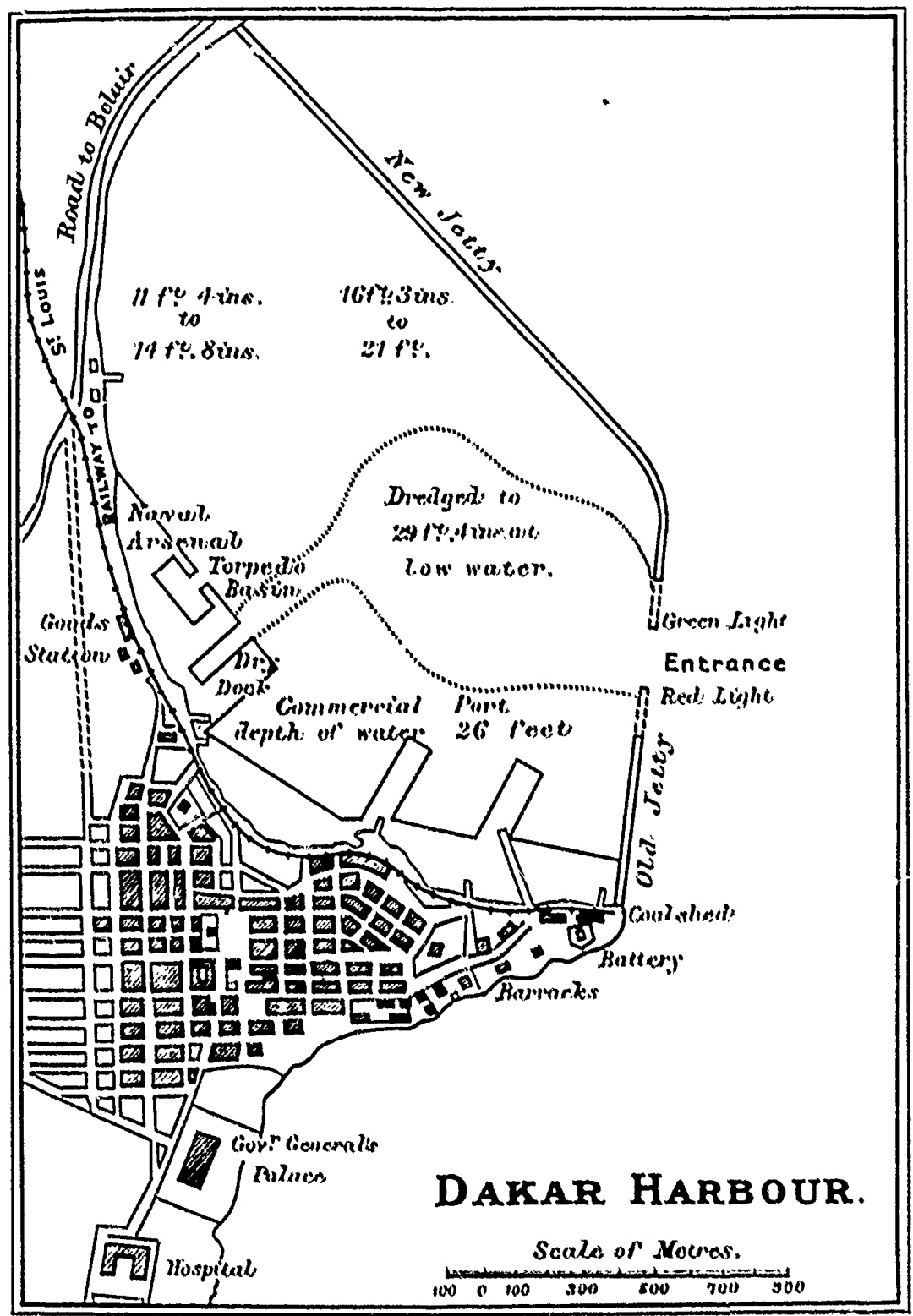

Fuente: House of Commons Parliamentary Papers Online. DCR, No. 3543 (1906) 
Sin embargo, como se señaló anteriormente, el segundo programa de modernización portuaria en Dakar comenzó a desarrollarse en la década de 1920, con el establecimiento -como se expondrá más adelante- del Consejo Portuario, así como la ampliación de los muelles y la decisiva introducción de bocas de suministro de combustibles líquidos. Esta segunda generación de reformas se contextualiza en el marco de inversiones proyectadas por el Ministerio de Colonias encabezado por el Ministro Albert Sarraut en 1921 que planteaba la inversión masiva en infraestructuras para incrementar la actividad económica de las colonias en beneficio del interés metropolitano y la reactivación económica de la Francia de posguerra ${ }^{18}$. En el puerto de Dakar, las aportaciones del Plan Sarraut podrían resumirse en la tímida apertura del mercado del suministro de combustibles líquidos que requerían obras importantes como tanques, bocas de suministro y un complejo sistema de tuberías y canalizaciones. Las primeras bocas de suministro de fuel-oil se instalaron en el dique norte entre 1924 y 1925, dónde la profundidad de los fondos permitía el movimiento y atraque de los buques más grandes. ${ }^{19}$ Esta década contempló la expansión de numerosas empresas petroleras a lo largo de los principales puertos oceánicos de la costa occidental africana. En estos puertos, algunos nombres como Shell, Vacuum Oil Company, Texaco o British Petroleum comenzaron a ser familiares. No obstante, la entrada de estas empresas en Dakar se retrasó hasta 1929 debido a las presiones ejercidas por parte del lobby anglo-francés formado por las empresas carboneras y de suministro de fuel en Dakar para evitar la entrada de nuevos competidores en un mercado de fuerte carácter oligopólico. ${ }^{20}$

Por otra parte, la década de 1920 supuso la introducción de algunos equipamiento modernos en el puerto como nuevas grúas eléctricas para la carga de mercancías pesadas como coches, materiales de construcción, maquinarías, etc. Para los trabajos de estiba siguió utilizándose la mano de obra africana de manera intensiva, debido al reducido coste salarial que suponían para las empresas portuarias. En cualquier caso, el Plan Sarraut que tenía una proyección de diez años quedó paralizado por la crisis financiera internacional de 1929, que provocó una grave crisis comercial en África Occidental. El descenso de la demanda de materias primas coloniales en los núcleos industriales europeos así como el hundimiento de los precios provocaron una crisis sin

\footnotetext{
${ }^{18}$ Coquery-Vidrovitch, Catherine (1979). "Colonisation ou impérialisme : la politique africaine de la France entre les deux guerres", Le Mouvement Social, No.107, p. 51-76.

${ }_{19}$ PRO. Ministry of Transports, MT23/680/1 (1916).

20 Journal Officiel de l'AOF. 16 de Mayo de 1924 y 20 de Junio de 1925.
} 
precedentes en las estructuras agrarias del AOF, orientadas hacia los monocultivos de exportación $^{21}$. La crisis de 1929 trató de combatirse por parte de las autoridades coloniales en el AOF con un plan de choque fundamentado en la concentración de actividad económica en una serie de núcleos costeros. Dakar se configuró como uno de esos núcleos fundamentales, bajo el mandato del Gobernador Jules Brevié (1930-36) quién impulsó un proceso de reformas profundas en las infraestructuras portuarias en Dakar. La extensión del sistema Decauville para incrementar la productividad y eficiencia de Dakar supuso que el viejo centro exportador de Rufisque perdiera competitividad y que las principales empresas imperiales trasladaran sus oficinas hacia Dakar de manera definitiva. La mejora de las conexiones intermodales en Dakar se añadió a unos costes totales inferiores que hacían la escala en el gran puerto senegalés mucho más atractiva a los armadores y capitanes de los buques ${ }^{22}$. Entre 1931 y 1933 se proyectaron diferentes obras de mejora en Dakar, cuyo objetivo fue la ampliación de los terraplenes anexos al puerto dónde podrían construirse nuevos almacenes orientados sobre todo al almacenamiento de cacahuetes en la zona norte. Por otra parte, se culminó la instalación de las bocas de suministro de combustibles líquidos en un muelle de fuel-oil en el dique norte debido al notable incremento de la demanda por parte de los buques en tránsito. Este proyecto en 1931 que se complementaba con el aumento de la profundidad en toda la zona abrigada a 10 metros supuso una inversión de 5,7 millones de francos ${ }^{23}$. En última instancia, estos proyectos buscaban incrementar la competitividad inter-portuaria de Dakar frente a los puertos canarios para absorber tráficos de escala. Las obras de mejora en el puerto de Las Palmas desarrolladas en 1932 requerían de una respuesta eficaz por parte de las autoridades portuarias en Dakar en lo que se refería a las funciones de escala. Como puede observarse en los siguientes gráficos, los tráficos portuarios en Dakar se incrementaron notablemente en cada una de las reformas referidas entre 1900 y 1939, atendiendo a diferentes causas internas y externas como ha sido señalado (Gráficos 2 y 3). Cabe destacar el pico alcanzado en 1936 como consecuencia entre otras cuestiones del estallido de la Guerra Civil española y el desvío de los tráficos comerciales desde los puertos canarios hacia Dakar, que elevó notablemente la actividad comercial en el puerto senegalés.

${ }^{21}$ Daumalin, Xavier (1992). "Marseille, I'Ouest Africain et la Crise". En M. Courdurie et J.L. Miege (eds.) Marseille Colonial face a la crise de 1929 (Chambre de Commerce et d'Industrie Marseille-Provence), p.198.

${ }_{22}$ Wittlesey, Derwent (1941). "Dakar and other Cape Verde settlements". In Geographical Review, Vol.31-4, American Geographical Society, p. 618.

${ }^{23}$ Journal Officiel de I'AOF. 30 de Marzo de 1929. 
Gráfico 2. Tráfico portuario en Dakar (1906-1939) (Buques entrados y TRB)

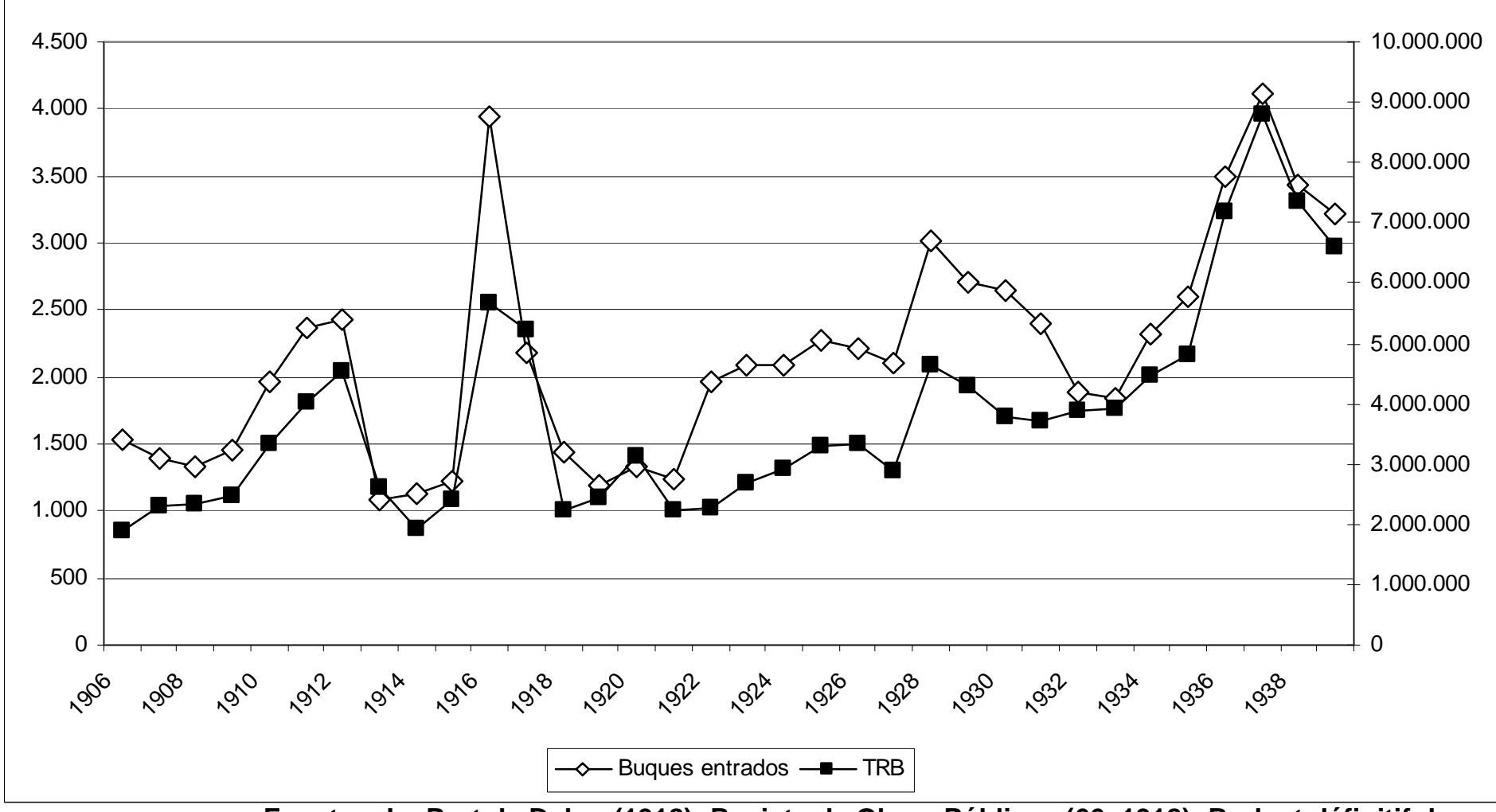

Fuentes: Le Port de Dakar (1918); Revista de Obras Públicas $(66,1918)$; Budget définitif du port de commerce de Dakar (1911-1940); ANSOM (Agefom 83/363; TP, Carton 568); ANS 2G7-34; Wittlesey (1941); Dakar, Station Service Atlantique (1949), Morazé (1936)

Gráfico 2: Tráfico comercial en el puerto de Dakar (1900-1939) (en toneladas)

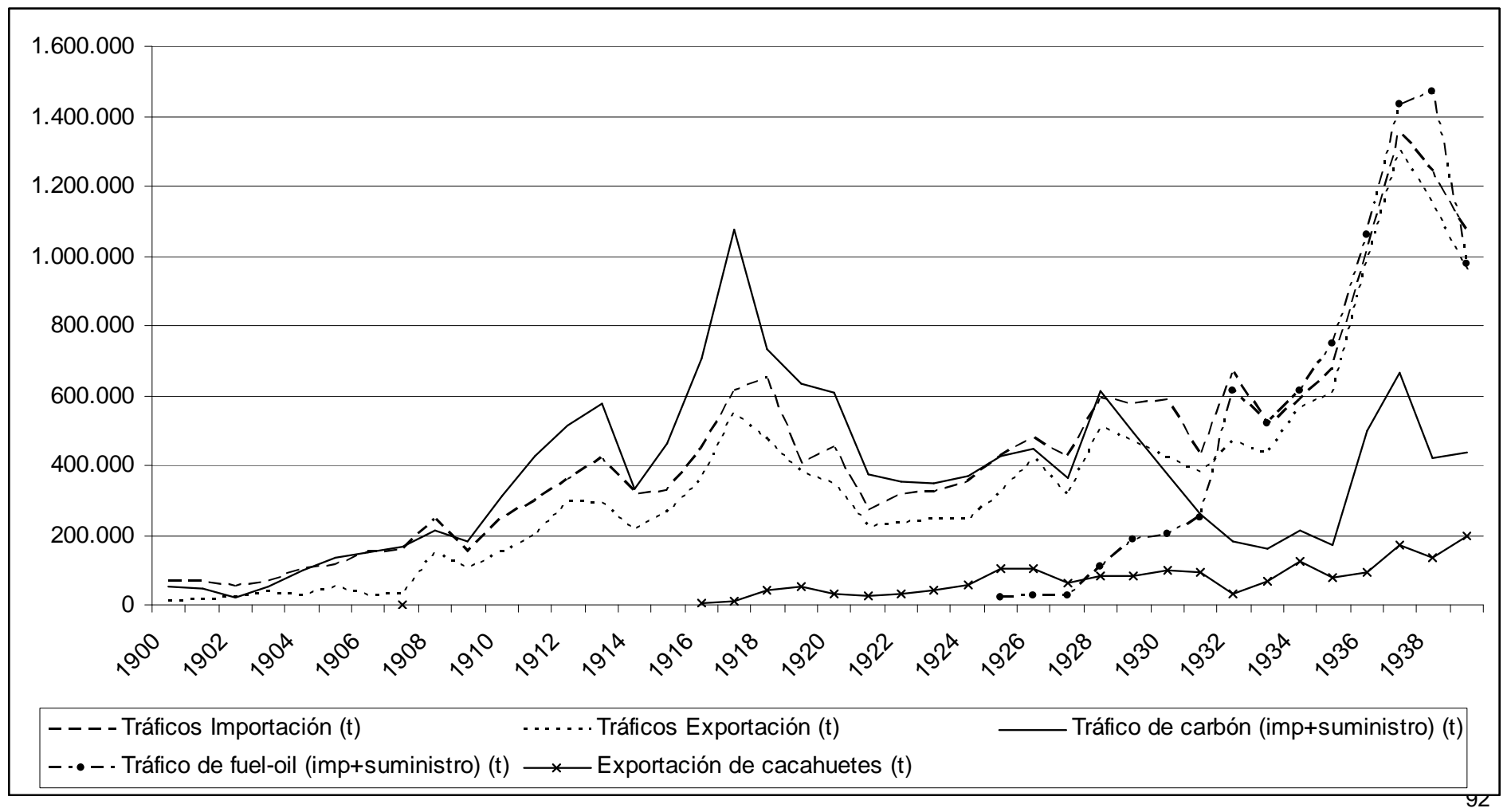


Fuentes: Le Port de Dakar (1918); Revista de Obras Públicas $(66,1918)$; Budget définitif du port de commerce de Dakar (1911-1940); ANSOM (Agefom 83/363; TP, Carton 568); ANS 2G7-34; Wittlesey (1941); Dakar, Station Service Atlantique (1949), Morazé (1936)

\section{La reforma de la administración portuaria en Dakar (1910-1929)}

Los avances en la industria naval que se desarrollaron en el último tercio del siglo XIX incrementó la actividad en los puertos de manera notable. El incremento en la demanda de servicios portuarios, especialmente en la consignación y suministro de buques configuró un sector empresarial portuario dinámico y conectado con otros sectores industriales y comerciales. De este modo, la comunidad empresarial portuaria se incrementó con una mayor presencia e importancia de agencias consignatarias, comisionistas, corredores de seguros, intermediarios, etc. ${ }^{24}$ El papel tradicional desempeñado por los capitanes en la actividad marítima (fletes, seguros, contratos, suministros) se reemplazó progresivamente por un conglomerado de agentes económicos y empresas de servicios portuarios integrados en el proceso integral de modernización de las infraestructuras portuarias y la industria naval a escala global. Estos cambios en los agentes económicos se complementaron además con las reformas institucionales desarrolladas en los puertos para regular el incremento de la actividad portuaria, que proporcionaba además unos interesantes beneficios en forma de tasas portuarias, impuestos, arbitrios y otros ingresos a favor de las administraciones públicas. El contexto de crecimiento comercial encarnado en la expansión de la actividad marítima durante este periodo hizo necesario una mayor ordenación de las políticas de administración portuaria a través del establecimiento de reglamentos de funcionamiento de los puertos claros y eficientes, además de la creación de instituciones civiles para ordenar y regular la actividad portuaria. Este proceso se culminó en las dos primeras décadas del siglo XX cuando se crearon las autoridades portuarias en los principales puertos comerciales en África Occidental, proceso de similar desarrollo a escala global.

En Dakar, las primeras instituciones civiles que regularon la actividad portuaria se crearon en 1910, coincidiendo con la inauguración oficial del puerto de comercio. Anteriormente, las infraestructuras portuarias habían sido administradas por el gobierno del AOF y la marina francesa, quedando la función comercial supeditada a la militar. En ese año el puerto comercial -y con él sus instituciones- se desligaron de la administración

${ }^{24}$ Esto ha sido descrito por Suárez Bosa, M. (2003) y González Lebrero (1989). 
militar, conformándose una jerarquía civil que se culminó en 1920 con la creación del Consejo del Puerto formado por autoridades políticas y empresariales, como será expuesto más adelante.

En lo que se refiere al modelo general de administración portuaria, Dakar respondería en líneas generales al tipo Landlord durante el periodo colonial (tabla 1). La administración del puerto se sustentaba en un sistema mixto en el que el sector privado y las instituciones públicas se distribuían sus áreas de influencia, pero operando en permanente interacción. En términos generales la iniciativa privada y el sector público operaban bajo parámetros de incremento de la competitividad intra-portuaria (sector público), inter-portuaria (sector privado-público) y la obtención de beneficios (sector privado). Estas tres áreas de intervención se sostenían en el interés por atraer los tráficos marítimos desde otros puertos para lo cual se requería eficacia y competitividad en los servicios: tiempos de espera reducidos, coste de los combustibles, tasas portuarias, políticas arancelarias, existencia de fletes de retorno, cualificación de la mano de obra, trámites burocráticos portuarios, sector terciario competitivo, buenas condiciones de las instalaciones, etc. En este sentido, la combinación de estos factores y su mayor o menor competitividad ejercen una influencia decisiva sobre la actividad portuaria ${ }^{25}$. En este modelo de gestión portuaria, las instituciones públicas velan por el desarrollo de las infraestructuras y su mantenimiento general. El sector público realiza los mayores desembolsos en trabajos generales como mantenimiento de muelles, dragados y limpieza de los fondos, extensión de diques, iluminación pero también coordina las tareas de pilotaje, revisión y control aduanero, inspecciones sanitarias, etc. Las instituciones públicas realizan también concesiones administrativas de terrenos anexos al puerto bajo determinadas condiciones a las empresas de servicios portuarios para su explotación efectiva, lo que las sitúa en una posición ventajosa con respecto a otras empresas que no gozaban de esa concesión. Las empresas concesionarias abonarían un canon a las autoridades portuarias y se comprometerían a favorecer el desarrollo de la actividad marítima y la creación de puestos de trabajo. Finalmente, las autoridades públicas en Dakar recibían los ingresos de las tasas portuarias, arbitrios y concesiones que servían para la financiación del presupuesto del puerto, que era independiente del presupuesto general del AOF desde 1910.

\footnotetext{
${ }^{25}$ Jansson, J. and Shneerson, D. (1982). Port Economics, Cambridge: MIT Press.
} 
Tabla 1 Modelo de Administración portuaria en Dakar (1910-1929)

\begin{tabular}{cc}
\hline Ámbito de actuación & Administración \\
\hline Infraestructuras (construcción, mantenimiento y mejoras) & Pública \\
Servicios portuarios, explotación del Puerto (carboneo, astilleros, & Privada \\
comercio) & \\
Promoción y competencia inter-portuaria (tasas, arbitrios, precios de & Mixta \\
servicios portuarios) & \\
\hline
\end{tabular}

\section{Fuente: Castillo-Hidalgo (2012a)}

Por otra parte, es importante indicar que la administración colonial no se planteó de manera sistemática el establecimiento de una autoridad portuaria civil en Dakar hasta la inauguración del puerto comercial en 1910, pese a que las políticas metropolitanas desde la presidencia de Mac-Mahon (1873-79) se orientaron hacia una configuración civilempresarial de las instituciones portuarias, dónde las Cámaras de Comercio jugaron un papel destacado. Las autoridades coloniales en el AOF se mostraron más interesadas en mantener unos stocks de carbón aceptables en Dakar para servir a la navegación internacional y sobre todo a la marina francesa como pudo observarse en el acuerdo de abril 1884 entre el Gobierno metropolitano y Méssageries Maritimes para el mantenimiento de un stock permanente de carbón. Los cambios más profundos e importantes desde el punto de vista de la administración portuaria se produjeron en la década de 1920. La influencia de la administración militar fue reemplazada progresivamente por funcionarios civiles. Antes de la reforma institucional de 1920, el puerto estaba bajo la administración directa del Secretario Colonial de Obras Públicas, quién respondía ante el Gobernador General del AOF y el Ministerio de las Colonias. Como capitán del puerto, el Secretario Colonial organizaba la entrada de buques en el puerto y su amarre, regulaba el sistema de iluminación y balizas en el puerto y preparaba los protocolos de emergencia y rescate que pudiesen ocurrir en la Bahía de Dakar. ${ }^{26}$

No obstante, las funciones del capitán del puerto se restringían a labores logísticas, sin que ninguna autoridad velara por el buen funcionamiento de la actividad comercial de modo independiente, lo que lastraba el desarrollo eficiente de la actividad económica en Dakar. En 1910 el presidente de la Cámara de Comercio de Dakar (establecida en 1888),

${ }^{26}$ ANSOM. TP. Carton 507. Dossier 6. 
M.Formeraux envió una notificación oficial al gobierno del AOF en la que expresaba las reclamaciones de la comunidad empresarial en la ciudad-puerto senegalesa por constituir una administración portuaria civil y con una normativa clara y transparente que pudiera ser fácilmente interpretable por los capitanes de los buques que recalaran en el puerto. El presidente de la Cámara de Comercio señalaba además la importancia capital que debía jugar la institución empresarial y sus representados en la futura administración portuaria. ${ }^{27}$ Las demandas de la Cámara de Comercio fueron aceptadas después de una década, dado que el estallido de la Gran Guerra frenó cualquier avance en lo que se refería a la administración civil del puerto. Las necesidades militares y la primacía de la defensa desde 1914 interrumpieron los tímidos progresos que se estaban desarrollando en la administración portuaria en Dakar. En el periodo 1914-18, la gestión del puerto quedó en manos del Gobierno Federal y el Ministerio de Marina quiénes organizaron la actividad portuaria bajo criterios militares, continuando la percepción de tasas portuarias y organizando la construcción de infraestructuras portuarias como los nuevos depósitos de carbón en 1916-17.

Finalizada la Guerra, la administración portuaria en Dakar experimentó la reforma más importante de su historia hasta esa fecha. El 18 de octubre de 1920 el puerto comercial de Dakar se separó definitivamente del Almirantazgo francés y se adscribió enteramente al Gobierno Federal del AOF, conformándose el Consejo del Puerto en el que la Cámara de Comercio iba a jugar un papel fundamental. El capitán del puerto fue reemplazado por un Director del puerto nombrado por el Gobernador del AOF. El cargo ocupado generalmente por un ingeniero especializado tenía una duración de cinco años y le otorgaba potestad sobre el personal dependiente de la administración pública en el puerto. Este cargo debía rendir cuentas al Consejo Portuario, que era el órgano consultivo, creado también en 1920 como órgano de representación de la comunidad portuaria en Dakar. El Consejo elaboraba planes estratégicos de desarrollo de la actividad económica en Dakar, funcionando como un elemento esencial de la administración portuaria puesto que era el organismo encargado en plantear, discutir y establecer las tasas y arbitrios, enmendar los presupuestos del puerto y proyectar mejoras e intervenciones en las infraestructuras. El Consejo presentaba además proyectos al Gobierno Federal en el caso de grandes obras públicas que sobrepasaran la capacidad presupuestaria del puerto, para solicitar la autorización para contratar préstamos. En

27 ANSOM 14 MIOM/1455. Informe sobre la gestión del Puerto remitido al Gobierno Federal del AOF, 3/10/1910. 
cualquier caso, el Consejo actuaba también como un grupo de presión puesto que la mayor parte de sus miembros eran representantes de las principales empresas portuarias y comerciales. Diez miembros del consejo eran agentes de las empresas de navegación, comercio e industria de Dakar, mientras que el resto estaba compuesto por el Secretario de Finanzas del AOF, el Director del puerto, el Gobernador General del AOF y un representante de los trabajadores del puerto nombrado por el propio Director. ${ }^{28}$

A mediados de la década, el organigrama del puerto se mejoró bajo parámetros basados en una organización especializada del trabajo en diferentes áreas que se mantuvo prácticamente inalterable hasta finales de la década de 1940. Esto se plasmó en la creación de una gran estructura dividida en dos departamentos superiores. El primero de ellos tenía como función la explotación económica del puerto, organizando el cobro de arbitrios y tasas, el pago de las nóminas y salarios de los trabajadores portuarios así como la organización de proyectos mecánicos y de ingeniería destinados a la mejora de las infraestructuras portuarias. Este departamento operaba en coordinación con los Servicios Económicos del AOF que velaban por el cobro de los impuestos aduaneros. Por otra parte, el otro gran departamento que se desarrolló en la década de 1920 fue el de mantenimiento diario y operaciones corrientes. En este departamento se incorporaron la mayor parte de trabajadores africanos de más baja cualificación al servicio de la administración portuaria. Esta sección incorporó también los servicios de limpieza y sanidad del puerto, creados en Diciembre de 1928 que velaban por el buen cumplimiento de las condiciones sanitarias e higiénicas en los buques y las instalaciones portuarias. Además, este departamento de funcionamiento ordinario regulaba el cuerpo de policía portuaria así como los jornaleros empleados, el personal de mantenimiento y los trabajadores y marinos encargados del pilotaje y rescate marítimo. Finalmente, en la década de 1930 se incorporó el servicio de energía y abastecimiento de agua y electricidad en Dakar bajo administración de este departamento, que operaba en coordinación con el Ayuntamiento de Dakar, que comenzaba a desarrollar el tendido eléctrico en la ciudad.

\footnotetext{
${ }^{28}$ El 31 de Mayo de 1950 se aprobó una modificación del sistema de electores del Consejo Portuario. El número de representantes procedentes de la Cámara de Comercio se elevó de 10 a 11. ANS. Cámara de Comercio de Dakar. Section 09. 00485-26/23.
} 


\section{Conclusiones}

En este artículo se ha mostrado cómo el puerto de Dakar atravesó diferentes etapas de modernización de sus infraestructuras y administración ante el crecimiento del comercio regional, los avances tecnológicos en el campo de los transportes modernos y la expansión europea en África Occidental. Este proceso de modernización se desarrolló en diferentes aspectos que abarcaban desde las propias infraestructuras (diques, muelles, dragados, iluminación) a los modelos de gestión portuaria (consejos portuarios, administraciones civiles, interacción entre el sector privado-público). Como ha sido reseñado, el puerto de Dakar se convirtió en un eje económico y político fundamental en Senegal y el conjunto del AOF, concentrando la inversión pública y privada. Dakar impulsó el proceso de introducción de Senegal y el AOF en las dinámicas económicas de la globalización a través de la extensión de los cultivos de exportación y el desarrollo de las redes ferroviarias que conectaron los centros agrícolas de interior con el Gateway atlántico. El puerto de Dakar mejoró sus infraestructuras y las adaptó para servir de un modo más efectivo a los intereses metropolitanos, incrementando la cantidad y la variedad de las exportaciones de los territorios coloniales. En suma, el puerto de Dakar fue el elemento esencial que favoreció la introducción de estas regiones en el mercado global de commodities, alterando profundamente las estructuras económicas, políticas y sociales preexistentes.

\section{Referencias Bibliográficas}

Bird, James (1971). Seaports and seaport terminals, London: Hutchinson University Library.

Bonin, Hubert (1987). C.F.A.O. Cent ans de compétition, Paris : Economica.

Brooks, G. E. (1975). "Consequences of the Commercializartion of Peanuts in West Africa, 1830-70", The Journal of African History, XVI-1, p. 29-54.

Castillo Hidalgo, Daniel (2012a). Tendiendo Puentes: Dakar y la configuración de la red portuaria de África Occidental. Evolución y análisis de una comunidad portuaria (18571957). Unpublished Doctoral Thesis, University of Las Palmas de Gran Canaria.

Castillo Hidalgo, Daniel (2012b): "Buques, ferrocarriles y cacahuetes. Modernización de las infraestructuras en el puerto de Dakar y la introducción de Senegal en los mercados internacionales (1857-1936)", Transportes, Servicios y Telecomunicaciones, XXIII, p. 140164. 
Charpy, Jacques (2007). Dakar. Naissance d'une Métropole, Dakar : Editions Les Portes du Large.

Charpy, Jacques (2011). "Aux origines du port de Dakar ”. In Outre-Mers, T.99-370/371, p. 301-317.

Coquery-Vidrovitch, Catherine (1979). "Colonisation ou impérialisme : la politique africaine de la France entre les deux guerres". In Le Mouvement Social, No.107, p. 51-76.

Daumalin, Xavier (1992). "Marseille, I'Ouest Africain et la Crise". In M. Courdurie y J.L. Miege (eds.) Marseille Colonial face a la crise de 1929, Marseille : Chambre de Commerce et d'Industrie Marseille-Provence, p. 168-218.

Debrie, Jean (2010). "From colonization to national territories in continental West Africa : the historical geography of a transport infrastructure network". In Journal of Transport Geography, 18, p. 292-300.

Debrie, Jean (2012). "The West African port system: global insertion and regional particularities”, EchoGéo, 20 (avril/juin).

González Lebrero, Rodolfo (1989). El agente consignatario de buques en España, Barcelona: Librería Bosch.

Hopkins, A.G. (1973). An Economic History of West Africa, New York: Columbia University Press.

Jansson, J., and Shneerson, D. (1982). Port Economics, Cambridge: MIT Press.

Le Port de Dakar (1918). Dakar : Grande Imprimerie Africaine.

Les Colonies Françaises (1857). La Revue Coloniale, Paris: Imprimerie et Librairie Administrative de Paul Dupont.

Marnot, Bruno (1999). "La politique des ports maritimes en France de 1860 à 1920", Histoire, économie et société. $18: 3$, p. 648-652.

Moitt, Bernard (1989): "Slavery and Emancipation in Senegal's Peanut Basin: The Nineteenth and Twentieth Centuries", The International Journal of African Historical Studies, XXII-1, p. 27-50.

Morazé, Charles (1936). “Dakar”, Annales de Géographie, Vol.45-258, p. 607-631.

Ndao, Mor (2009). Le ravitaillement de Dakar de 1914 à 1945, Paris: L'HarmattanSénégal.

Olukoju, Ayodeji (2004). The Liverpool of West Africa, New Jersey: Africa World Press.

Peterec, Richard J. (1967). Dakar and West African Economic Development, New York: Columbia University Press. 
Polónia, Amélia (2010). "European seaports in the Early Modern Age: concepts, methodology and models of analysis", Cahiers de la Méditerranée, 80, p. 17-39.

Rodney, Walter (1971). How Europe Underdeveloped Africa, Dakar: Pambazuuka Press, [2011].

Seck, Assane (1970). Dakar, Métropole Ouest-Africaine, Dakar : IFAN.

Suárez Bosa, Miguel (2003). Llave de la Fortuna. Instituciones y Organización del trabajo en el Puerto de Las Palmas, 1883-1990, Las Palmas: Fundación Caja Rural.

Thomas, Benjamin (1957) "Railways and Ports in French West Africa", Economic Geography, XXXIII-1, p. 1-15.

Wittlesey, Derwent (1941). "Dakar and other Cape Verde settlements", Geographical Review, Vol. 31-4, American Geographical Society, p. 609-638.

Recebido para publicação em 31 de julho de 2013.

Aprovado para publicação em 07 de outubro de 2013. 